\title{
EXPRESSION DE L'OPINION ET TRANSMISSION DES SAVOIRS DANS LA PRESSE QUOTIDIENNE
}

\author{
Annik Dubied ${ }^{1}$
}

Le questionnement qui a initié cet article m'a été suggéré par mes travaux précédents. J'ai longtemps travaillé sur le fait divers, un genre réputé pour ne nécessiter "aucune connaissance préalable", puisqu'il ne requiert "rien de plus" que la mise en service partielle des connaissances élémentaires qui font partie de l'encyclopédie (au sens d'Umberto $\mathrm{Eco}^{2}$ ) de tout un chacun, et qui permettent de décoder et de remplir les blancs que tout message contient.

M'interroger, au-delà du fait divers, sur des genres qui, pour leur part, reposent en tout cas partiellement sur des connaissances additionnelles, spécifiques à un champ particulier, et qui dépassent largement l'encyclopédie de base citée ci-dessus, m'a paru une intéressante mise en perspective. Car en effet, l'éditorial ou le commentaire, articles courts, disent une opinion sur un sujet qui, en toute logique, doit être connu pour que leur prise de position soit comprise et, éven-

1 Maître-assistante à l'Université de Genève (Sociologie et DEA en "Communication et médias").

2 Chez Eco (voir notamment Lector in fabula, Paris, Grasset et Fasquelle, 1985 (1979)), le concept fonctionne pour les récits ; il peut néanmoins être heureusement exporté, dans le cas qui m'occupe. 
tuellement, acceptée. Cette expression de l'opinion est donc intimement liée à une mise au point ou une négociation des savoirs (formels et informels) qui l'autorisent.

Je me suis par conséquent demandé comment les journaux opéraient pour mettre en place ou rappeler la base de connaissances sur laquelle repose leur transmission de l'opinion? Quels sont les moyens de l'expression de l'opinion rédactionnelle, et comment celleci gère-t-elle la "valeur ajoutée" de connaissances qu'elle doit se donner ? Je parlerai donc du savoir "en creux", sans jamais me pencher directement sur lui, mais en parlant des modes de renvoi qui $y$ mènent et du statut qui lui est attribué.

\section{Un cadre : la communication politique}

L'expression de l'opinion dans la presse fait partie d'un cadre qui la dépasse : la communication politique, qui implique une multitude d'acteurs et un jeu complexe d'échanges, de négociations et de coups de force. En toile de fond de ce cadre global, l'espace public, un des enjeux qu'évoquait l'appel à communication du colloque, et les liens que la presse entretient avec la citoyenneté.

J'ai choisi de prélever un échantillon de cette expression, un échantillon partiel et limité. J'ai retenu l'expression de l'opinion rédactionnelle dans la presse à travers deux genres spécifiquement consacrés à elle : l'éditorial et le commentaire. A ce choix, deux raisons : en premier lieu, la nécessité de limiter mes recherches (pour qu'elles restent raisonnables) à une portion du système très complexe ci-dessus mis en évidence, et à l'un de ses acteurs -les rédactions et "leur" opinion. Ensuite, il s'agissait de rendre justice aux potentiels rédactionnels (interfaces, moyens sémiotiques) que seuls des membres actifs d'un journal sont à même de solliciter.

Le cadre du problème que j'évoque aujourd'hui est fondamental, puisque la transmission du savoir y est, j'espère le montrer, un enjeu de persuasion, de réussite et de pouvoir. A travers lui, par lui et en lui se forgent et se transmettent les opinions.

Le savoir est le ciment des relations entre interlocuteurs, qui autorise la prise de position du locuteur ${ }^{1}$.

1 M. Souchard, Le discours de presse. L'image des syndicats au Québec (19821983), Montréal, Le Préambule, 1989, p. 117. 
Mon entrée est donc limitée à une portion du contexte décrit. C'est par l'expression de l'opinion rédactionnelle que je tente de repérer les diverses formes de transmission du savoir.

\section{Quelques représentants de la presse régionale suisse-romande}

J'ai relevé les éditoriaux et les commentaires sur une semaine (du 18 au 26 novembre 2000), qui se trouvait être la semaine précédant une importante votation populaire en Suisse. Une partie des articles (moins importante que je ne le prévoyais, toutefois) était donc consacrée aux différents objets de cette votation.

J'ai choisi trois journaux régionaux: Le Courrier (genevois, explicitement de gauche, très engagé), Le Nouvelliste (valaisan, conservateur) et La Tribune de Genève (genevois, populaire/se voulant "neutre", appartenant au groupe Edipresse). A ce corpus, j'ai ajouté quelques pointages opérés dans Le Quotidien jurassien (jurassien, comme son nom l'indique, et conservateur) sur une période plus étendue. Ce choix de journaux avait à mes yeux l'intérêt non seulement de mettre en évidence la richesse, la pluralité et la diversité de la presse régionale suisse ${ }^{1}$, mais aussi de mettre en perspective des journaux qui fonctionnent encore, partiellement et de manière plus ou moins accentuée, sur le modèle d'une presse engagée, proche de la presse d'opinion aujourd'hui disparue.

Ma récolte a abouti à un corpus d'environ cinquante articles de presse, que j'ai analysés en m'interrogeant sur leur mise en page, sur les types de renvois intertextuels qu'ils opéraient, sur leurs implicites, sur leur énonciation et sur le degré de complexité de leur lexique.

Précisons que les journalistes qui signent ces articles ne sont pas des spécialistes de la vulgarisation scientifique ou de la transmission des connaissances. Ils sont plutôt, en principe, des rédacteurs confirmés, susceptibles non plus vraiment de transmettre l'opinion de l'organe de presse lui-même, mais d'incarner le sérieux, le doigté et le sens de l'à-propos de leur rédaction.

1 Les seuls véritables journaux de dimensions supra-régionale en Suisse romande, $L e$ Temps et Le Matin, sont trop différents pour donner lieu à une analyse comparative pertinente. 


\section{Expression de l'opinion et stratégies d'exposition du savoir}

\section{Les genres du commentaire}

L'éditorial et le commentaire font partie des genres journalistiques qui utilisent un mode d'expression qu'on pourrait qualifier d'impliqué et de subjectif. Ils sont les genres par excellence où s'exprime une opinion issue de la rédaction.

Tous les deux disposent de divers moyens pour se signaler : leur étiquette respective, d'abord, massivement présente dans le corpus. Leur typographie, ensuite, toujours très différente de celle du reste de la page. Divers moyens de mise en page, encore, qui les isolent et les distinguent, comme l'encadré ou un positionnement spécifique.

L'éditorial a beaucoup changé ces dernières années, mais reste un genre privilégié, qui apparaît, sinon en première page, du moins en seconde, dans tous les journaux étudiés. Parfois, il a perdu son nom pour devenir "Commentaire", même en une. Le commentaire au sens originel du terme s'allie pour sa part plus volontiers à un article au cœur du journal, pour revenir sur les faits exposés. L'un et l'autre semblent donc devoir employer des moyens différents de renvoyer à un savoir qui les accompagne et les autorise.

\section{Complexités lexicales et termes auto-déterminés}

Un bref survol des articles récoltés suffit pour constater que les éditoriaux comme les commentaires sont des articles au niveau de complexité élevé. Leur champ lexical est empreint d'une technicité que les rédacteurs sont manifestement peu soucieux d'euphémiser. Les textes sont en outre truffés de ce que Jenny Simonin appelle des termes "auto-déterminés" : ces noms propres, ces dates, que le cotexte n'explicite pas, renvoient à un hors-texte (contexte) dans lesquels ils doivent pouvoir être situés pour faire sens. Chose qui semble relativement simple lorsque le terme auto-déterminé est

1 J. Simonin, "Les repérages énonciatifs dans les textes de presse", La langue au ras du texte, Lille, PUL, 1984, pp. 133-203. Les termes sont alors "auto-déterminés" par rapport au texte et à la situation d'énonciation (ni déictiques, ni anaphoriques). 
"Yasser Arafat", mais qui l'est tout de suite moins lorsqu'il s'agit de l'OSCE (sans explication) ou du "7 novembre" (date de l'élection présidentielle américaine). Le co-texte est alors d'un secours très limité pour désambiguïser les termes en question.

Dès lors, on s'attend assez logiquement à ce que le journal s'emploie à offrir ces informations susceptibles de manquer à une frange importante du lectorat. Et c'est effectivement ce qui se passe souvent, par divers moyens.

\section{Techniques de mises en scène des savoirs et des opinions}

L'éditorial comme le commentaire sont par définition, on l'a dit, des articles courts, privilégiant un style efficace et soigné. Ils ne peuvent donc se permettre de consacrer plusieurs paragraphes à réexpliquer l'objet sur lequel ils se prononcent. D'où de nécessaires renvois à d'autres lieux qu'il est intéressant de détailler, même si leur recensement prend de prime abord des allures d'évidence... peut-être pas tout à fait aussi évidente qu'il y paraît.

La presse bénéficie de multiples ressources pour lier tout en les distinguant l'expression de l'opinion et la mise au point du savoir qui y est lié. Les progrès de la mise en page multiplient ces moyens. On dit souvent que le journal est un espace propice à l'expression de l'opinion ; du point de vue de la transmission des savoirs additionnels, en tout cas, cela s'avère tout à fait exact.

Le phénomène très moderne des hyper-structures apporte à ce problème une première solution. "Regroupement[s] d'articles et d'images graphiquement et sémantiquement liés, bornés par la double page"1, celles-ci permettent une lisibilité et une rapidité de lecture plus grandes en exploitant les ressources visuelles et de hiérarchisation qu'offre l'éclatement du sujet en petits sous-ensembles. Dans le corpus, outre plusieurs articles plus ou moins courts ou des infographies, elles accueillent volontiers un commentaire qui vient s'ajouter à un sujet traité en page intérieure. Elles offrent alors plusieurs entrées sur un même thème, et le commentaire constitue une des entrées possibles, se complétant facilement d'éléments plus descriptifs ou explicatifs qui le côtoient sur la page. L'opération de renvoi consiste

1 Voir notamment J.-M. ADAM et G. LUGRIN, "L'hyper-structure, un mode privilégié de présentation des événements scientifiques", Cahiers du CEDISCOR, n 6, 2000 (à paraître). 
alors en une forme de fléchage court (j'ignore quels termes techniques utiliseraient les cognitivistes pour désigner cette procédure) renvoyant à des éléments immédiatement voisins. A noter que le commentaire intervient alors systématiquement en bas et/ou à droite de l'ensemble que constitue l'hyper-structure, à la fin ou en ponctuation du reste de la matière.

Les éditoriaux procèdent eux aussi, souvent, à une forme de fléchage, mais qui s'opère alors sous forme de renvoi à d'autres pages. La une ou la deux renvoient à la page concernée, par simple mention au bas de l'article. Le fléchage s'opère alors, non plus au niveau d'une hyper-structure et de son unité supérieure, la double page, mais au niveau des rubriques, et de leur unité supérieure, le journal. Pour reprendre les termes profanes évoqués précédemment, on aurait alors ici un fléchage long.

Cas de figure atypique et pour le moins original, un des journaux du corpus, Le Courrier, procède pour sa part volontiers à des renvois en note de bas de page. Les scientifiques connaissent bien cette procédure, rare dans la presse, qui permet de rappeler rapidement des éléments essentiels. Au-delà de sa dimension relativement exceptionnelle dans ce cadre-là, ce procédé vient souligner le problème auquel les éditorialistes se frottent lorsqu'ils veulent exprimer leur opinion, et permet de comprendre la multiplication des démarches énumérées ici.

L'article renvoie parfois directement à un extérieur précis susceptible de renseigner sur l'objet traité : un autre numéro du quotidien, ou la petite brochure que reçoit tout citoyen suisse en droit de voter ${ }^{1}$ servent alors de références faciles à mobiliser.

Reste enfin le cas où aucun renvoi explicite n'est opéré, lorsque l'éditorial (et lui seul) s'exprime sur un fait d'actualité qui n'est pas évoqué dans le même numéro du journal. Le thème est alors en quelque sorte imposé par l'actualité, et il a fait l'objet, en principe, d'une large médiatisation. La technicité de ces textes "isolés" n'est pas moins grande que celle des autres articles du corpus, malgré l'absence d'explicitation d'un savoir additionnel. Ce cas de figure m'a beaucoup intriguée. Le lecteur moyen peut-il se passer de trouver la référence des termes techniques non-explicités ? Peut-il se passer d'un cadrage ? La médiatisation, dont le thème commenté a par ailleurs fait l'objet, est-elle suffisante pour éviter que tout autre mise au point du

1 Une brochure orientée puisqu'elle présente les objets soumis au vote en indiquant les "recommandations" du Conseil Fédéral, l'exécutif national. 
savoir ne soit opérée ? Sans doute tout ceci est-il partiellement vrai, mais le cas reste intriguant.

Le savoir auquel il est fait référence représente, comme dans les autres cas de figure, un niveau encyclopédique supérieur à celui que j'ai signalé en introduction à propos du fait divers. Son partage "universel" est plus que problématique. Il serait donc logique de le rappeler, de l'ajuster et, je l'ai déjà signalé, de le négocier (on se doute que sa présentation ne sera jamais "neutre", même si je ne me suis pas penchée sur cet aspect des choses) pour que la base de connaissances devienne plus ou moins commune à tous. C'est l'opération que j'ai observée plus haut à travers les multiples procédures de renvois ; c'est celle qui manque ici.

La meilleure réponse à apporter aux questions que suscite ce dernier cas de figure serait à l'évidence une étude des pratiques des usagers. Néanmoins, l' analyste des messages que je suis peut formuler l'une ou l'autre hypothèse, ou en tout cas signaler quelques observations qui y sont liées.

\section{"Le savoir comme idéologie"}

Il faut rappeler d'abord le rôle primordial de l'implicite dans l'actualisation d'un texte '. L'implicite est d'ailleurs lui aussi une forme de renvoi ou de fléchage, par les inférences qu'il requiert. Les allusions (souvent très techniques) à un savoir additionnel renvoient donc implicitement à une somme de connaissances, partagées ou non, et dont ces allusions dessinent les contours. A ce stade, l'idée développée par Maryse Souchard dans son livre sur le discours sur les syndicats au Québec paraît particulièrement éclairante. Dans un chapitre intitulé "Le savoir comme idéologie", elle observe que, dans le discours sur les syndicats (mais aussi dans les articles du présent corpus) :

(...) la persuasion passe quasi nécessairement par un lieu [un savoir, ajouterais-je, Ndl'A] commun où se retrouvent locuteur et récepteur, où ils s'entendent, d'une part ; [que] d'autre part,

1 Pour une définition de l'implicite, voir notamment Catherine KERBRATORECCHIONI, "Discours politique et manipulation: du bon usage des contenus implicites", Le discours politique, Lyon, PUL, 1984, pp. 213-227. 
ce lieu n'a pas besoin d'être obligatoirement exprimé, qu'il lui suffit d'être admis'.

Renvoyer à un savoir qu'on pose comme commun est donc déjà en soi une opération de persuasion. L'implicitation devient dans ce cas un adjuvant puissant, puisqu'on sous-entend que :

Ce qui est dit s'attribue alors à l'opinion commune (...) la persuasion passe par la doxa bien plus que par la position sociale de celui qui parle 2 .

La technicité des textes peut alors s'expliquer, au moins partiellement, par le fait qu'elle accentue le rôle de l'implicite, tout en soulignant le savoir du locuteur et en lui conférant, de fait, une part supplémentaire d'autorité. Car ce savoir posé par lui comme commun, il le possède, il le maîtrise et il en définit les contours.

\section{Énonciation et doxa}

L'énonciation des textes (ceux de Souchard, mais aussi une part signifiante de ceux qui font l'objet de cette analyse) vient renforcer ce renvoi à un monde de connaissances postulées communes. Sur ce point, il faut rappeler rapidement que le mode énonciatif privilégié par les nouvelles formes de commentaires dans la presse est inspiré du modèle anglo-saxon. La tendance est à l'euphémisation des prises de position et des modalisations du style ${ }^{3}$. Il minimise aussi la présence d'un véritable sujet de l'opinion (au profit de sa collectivisation et de son rapprochement avec l'opinion publique postulée), et multiplie en outre les opinions dans un même support de presse, voire sur une même page, la pluralité s'imposant d'autorité au sein du journal luimême ${ }^{4}$.

1 M. SOUCHARD, op. cit., p. 119.

2 Ibid., p. 157.

3 Cette tendance se vérifie dans les textes seulement, puisque, comme le notent Nicole Jufer et Thierry Herman ("L'éditorial, vitrine idéologique du journal ?", Semen, $\mathrm{n}^{\circ} 13,2001$, pp. 135-162), le péritexte reste pour sa part très subjectivisé.

4 Voir notamment A. DubIED et M. LITS, "L'éditorial, genre journalistique ou position discursive ?", Pratiques, $\mathrm{n}^{\circ}$ 94, juin 1997, pp. 49-61, et N. JUFER et Th. HERMAN, Ibid. 
Les "on" et les "nous" (ces "je" prudemment collectivisés, selon les termes de J. Simonin ${ }^{1}$ ) se multiplient en effet dans le corpus ${ }^{2}$, impliquant de manière détournée l'énonciataire dans le discours de l'énonciateur. Jamais, d'ailleurs, l'énonciataire n'est directement interpellé à la deuxième personne ("vous/tu") ; son implication n'est, elle non plus, jamais explicite. Elle va de pair avec une évocation orientée (une négociation) de ce fameux savoir postulé comme "commun". La multiplication des "nous", des "on", des modalités de l'énonciation (!, ?, adverbes) permet d'inscrire implicitement (on pourrait presque dire "insidieusement") l'énonciataire dans le texte, et attribue ce qui est dit à l'opinion commune. A côté de ces inclusions apparaît une multitude de formules du type "c'est... que...", qui viennent re-définir sans en avoir l'air la base de ce dont on parle. Comme dans cet article tiré du Nouvelliste, qui constitue un bon exemple des phénomènes que je viens de décrire (je souligne) :

\section{Courage, on vote}

(...) Ainsi va la vie politique dans notre démocratie directe, on nous oblige à trancher à la hache là où le scalpel s'impose.

La loi sur le personnel de la Confédération ne suscite pas d'angoisses : l'État reste un employeur sûr, le texte proposé reste en avance sur le secteur privé. Il n'y a pas là amorce d'une débâcle sociale et ce n'est pas le protectionnisme d'un statut qui sauvera la fonction publique.

L'initiative Denner, comme d'habitude, simplifie à outrance un problème complexe, $(. .$.

Où le choix se complique, c'est avec les deux initiatives sur l'AVS. Le fond est bon, c'est le moment qui est inopportun. Faciliter la retraite à partir de 62 ans de manière flexible et généralisée, voilà un progrès social qui semble évident. Mais entre le souhaitable et le possible il y a la marge... de deux milliards du prix à payer. L'AVS est une maison solide, à condition de ne pas la démonter. La prudence commande d'attendre la 11e révision, dans laquelle on nous promet la retraite flexible. Et les promesses... (...)

Le Nouvelliste, $23 / 11 / 00$

A noter le "comme d'habitude", efficace moyen de suggérer ou même d'affirmer que ce qui est dit là "coule de source", pour tout le

1 J. SiMONIN, op. cit.

2 A noter que dans Le Courrier, qui semble décidément vouloir se distinguer, les "on" sont fréquemment utilisés pour désigner la partie adverse. 
monde. Se multiplient de même les métaphores, qui elles aussi redisent les choses d'une autre manière. Renvois, donc, mais aussi négociation et re-définition de l'objet commenté, en compagnie d'un lecteur impliqué malgré lui dans ce savoir (im-)posé comme commun. On pourrait presque parler ici d'une forme d'encouragement à la centration $^{1}$, voire à la fusion. Tout indique que la transmission des savoirs devient alors un enjeu de négociations et de coups de force rentrés.

\section{La place du savoir dans l'argumentation}

Souchard va même plus loin lorsqu'elle observe que, dans ce genre de contexte, le savoir et sa mise au point quittent, dans le procédé argumentatif, la mise au point des prémisses pour se déplacer vers ce qui précède l'argumentation et la sous-tend.

Une étude ultérieure sur le sujet exploré ici nécessitera un approfondissement du travail sur l'implicite, une réflexion sur l'idée d'un savoir commun "idéologisé" et l'étude plus poussée de la place du savoir dans le raisonnement argumentatif. Il faudra étudier, aussi, la présentation du savoir lui-même lorsqu'il est explicité (ce que je n'ai pas pu faire ici).

J'espère néanmoins avoir montré que, en l'occurrence, le savoir (mélange de savoirs formels réactualisés et de savoirs informels) est enjeu de persuasion et, indirectement, de pouvoir. Il fait l'objet de négociations, et sa définition même est au cœur des enjeux. Sa mise en place a donc un rôle à jouer dans la compréhension de la communication politique, comme, je l'espère, le phénomène de l'expression de l'opinion dans la presse peut de son côté éclairer, par la bande, la problématique des savoirs formels et informels et de leur effectivité.

1 ... au sens où l'entendent par exemple J.-P. Meunier et D. PERAYA (Introduction aux théories de la communication, Bruxelles, De Boeck, 1993, p. 205), qui parlent d'un mouvement de repli autour d'une "entité dans laquelle chaque membre se reconnaît et à laquelle il s'identifie, resserrant du même coup ses liens avec les autres membres. Pour engendrer la cohésion, le centre doit lui-même manifester la plus grande cohésion". 